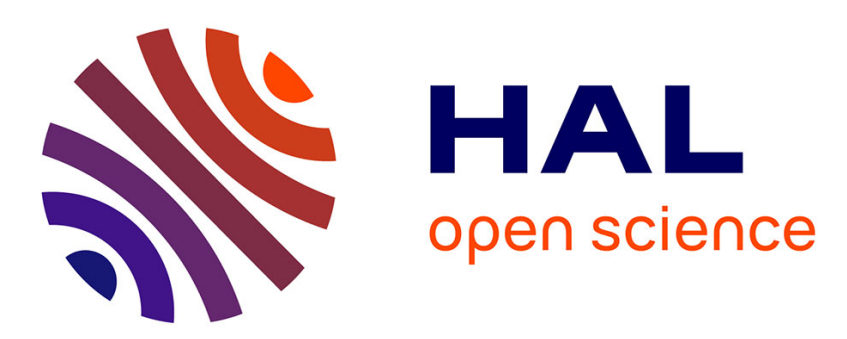

\title{
Comportement dynamique d'une composition explosive insensible
}

\author{
Chloé Rousseau, R. Belmas, P. Reynier
}

\section{To cite this version:}

Chloé Rousseau, R. Belmas, P. Reynier. Comportement dynamique d'une composition explosive insensible. Journal de Physique IV Proceedings, 1994, 04 (C8), pp.C8-421-C8-426. 10.1051/jp4:1994865 . jpa-00253426

\section{HAL Id: jpa-00253426 https://hal.science/jpa-00253426}

Submitted on 1 Jan 1994

HAL is a multi-disciplinary open access archive for the deposit and dissemination of scientific research documents, whether they are published or not. The documents may come from teaching and research institutions in France or abroad, or from public or private research centers.
L'archive ouverte pluridisciplinaire HAL, est destinée au dépôt et à la diffusion de documents scientifiques de niveau recherche, publiés ou non, émanant des établissements d'enseignement et de recherche français ou étrangers, des laboratoires publics ou privés. 


\section{Comportement dynamique d'une composition explosive insensible}

C. Rousseau, R. Belmas et P. Reynier

CEA, Centre d'Etudes du Ripault, BP. 16, 37260 Monts, France

Résumé : Nous présentons l'étude d'un modèle destiné à décrire le comportement thermomécanique d'une composition explosive pour des vitesses de déformation de l'ordre de $1000 \mathrm{~s}^{-1}$.

Les essais sont réalisés à des températures comprises entre 0 et $80^{\circ} \mathrm{C}$ sur une machine de compression rapide.

Le modèle est déduit de l'extrapolation d'une loi quasi-statique existante au domaine de vitesses de sollicitation considéré.

Une méthode analytique de modélisation intégrant la loi de comportement et le calcul des allers-retours d'ondes de contrainte dans l'échantillon est proposée pour simuler les essais. Les comparaisons calcul-expérience montrent que le modèle analytique est bien représentatif des résultats obtenus par essais.

Abstract : The aim of this study is to develop a model able to describe the dynamic behaviour of an explosive composition in the range of strain rates up to a few thousands per second.

The experiments were realized at temperatures between 0 and $80^{\circ} \mathrm{C}$, using a fast compression machine.

The behaviour model is deduced from mechanical tests at lower strain rates. This constitutive law suggests that an extrapolation is possible from the quasi-static to the dynamic domain.

We use an analytical method which includes the behaviour model and the stress waves paths in the sample to simulate the tests results.

A good agreement is obtained between dynamic experiments and modelling. 


\section{INTRODUCTION}

L'objectif de l'étude présentée ci-après est de vérifier l'aptitude d'une loi rhéologique (déterminée en quasi-statique pour des vitesses de déformation $\dot{\varepsilon}<$ quelques $10^{-4} \mathrm{~s}^{-1}$ ) à restituer le comportement d'un matériau agrégataire explosif dans le domaine du dynamique moyen ( $\dot{\varepsilon}<$ quelques $1000 \mathrm{~s}^{-1}$ ).

Les applications relèvent du domaine de la sécurité (chute, impact à faible vitesse), le but étant de prédire le comportement mécanique de structures intégrant l'explosif.

\section{MATERIAU ETUDIE}

Le matériau est obtenu par moulage à chaud sous pression de grains d'explosifs préalablement enrobés par un liant polymérique ( $97 \%$ de TATB pour $3 \%$ de liant). La densité $\rho$ de la composition explosive obtenue après pressage est de $1860 \mathrm{~kg} / \mathrm{m}^{3}$.

\section{MODELE RHEOLOGIOUE [1]}

Le comportement du matériau est très différent en traction et en compression, comme le montrent les résultats d'essais uniaxiaux obtenus à la température de $20^{\circ} \mathrm{C}$ pour une vitesse d'application de contrainte de $10 \mathrm{bar} / \mathrm{mn}$ (figure $\left.\mathrm{n}^{\circ} 1\right)$. Ce comportement est fortement non linéaire (surtout en compression).

Le matériau est, de plus, très sensible à la température et à la vitesse de sollicitation.

Dans le cas du comportement en compression uniaxiale, le modèle théologique reliant contraintes et déformations est établi par l'intermédiaire d'un module sécant $\mathrm{E}$ dépendant du temps, de la température et de l'endommagement, et reliant contraintes et déformations globales.

$$
\sigma=\mathrm{E}(\mathrm{t}, \theta, \beta) \cdot \varepsilon
$$

L'endommagement $\beta$ est défini comme le rapport de la contrainte $\sigma$ existante dans le matériau et de la contrainte maximale admissible pour les conditions de temps et températures considérées (figure $\mathrm{n}^{\circ} 2$ ).

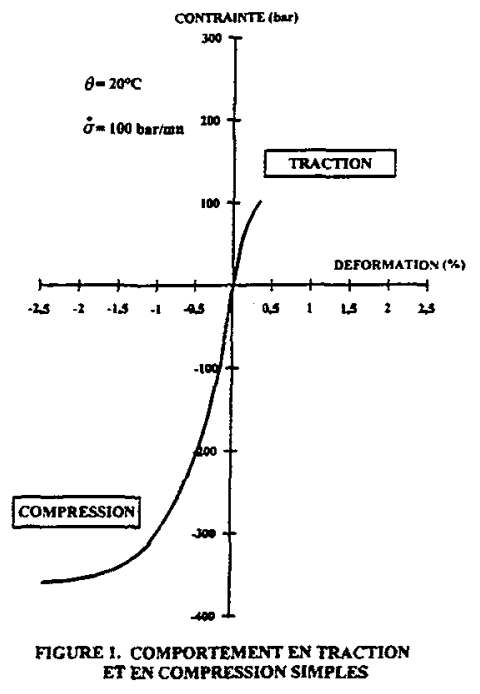

A partir d'essais effectués à différentes températures et vitesses d'application de contrainte, il est possible de déterminer la courbe maîtresse donnant l'évolution de la contrainte maximale en compression simple en fonction du temps réduit t/at $t_{\theta}$ pour une température de référence de $20^{\circ} \mathrm{C}$ (figure $\mathrm{n}^{\circ} 3$ ). 
Cette courbe est obtenue par l'intermédiaire d'une équivalence temps-température déterminée expérimentalement et présentée sur la figure $n^{\circ} 4$, où $\log a_{\theta}$ est un facteur de translation horizontal permettant, de manière classique, de raccorder les courbes mesurées pour les différentes températures d'essai. Le rapport $t$ at $_{\theta}$ est appelé temps réduit $t_{R}$.
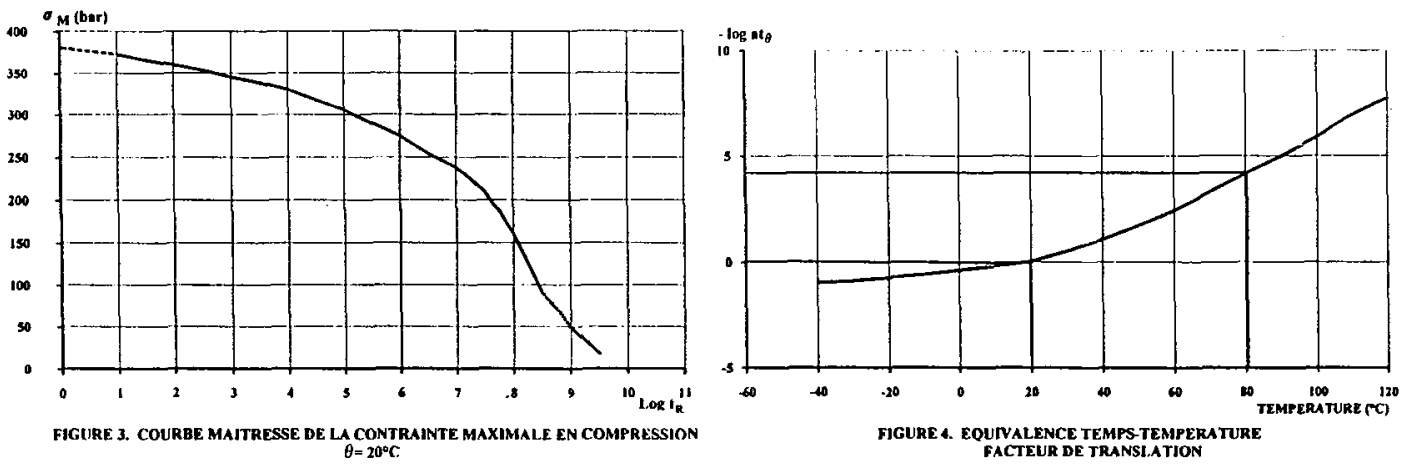

A partir de l'équivalence temps-tempétaluıc, it est possible de tracer également le module sécant uniaxial en fonction du temps réduit pour différents niveaux d'endommagement et une température de référence de $20^{\circ} \mathrm{C}$. On montre que ces courbes sont homothétiques. On peut définir alors, à l'aide d'un facteur multiplicatif FM lié à l'endommagement, une courbe maîtresse globale du module sécant uniaxial pour une valeur de référence $\beta=0,5$ (figure $n^{\circ} 5$ ). On a alors, pour une valeur quelconque de $\beta$ :

$$
\mathrm{E}_{(\beta)}=\mathrm{E}_{(0, \mathrm{~s})} \cdot \mathrm{FM}_{(\beta)}
$$

Les figures $\mathrm{n}^{\circ} 3$ et 5 montrent que, dans le cas des vitesses de déformation élevées (temps réduit faible), la contrainte maximale et le module sécant atteignent un palier. Dans ce domaine, le comportement du matériau est donc indépendant du temps et de la température, ce qui simplifie considérablement la modélisation rhéologique. En conséquence, aux vitesses de déformations élevées, le comportement en compression uniaxiale est décrit par la courbe unique contrainte-déformation présentée sur la figure $n^{\circ} 6$. La contrainte maximale $\sigma_{M}$ vaut 380 bar. Le module à l'origine Eo identifié à 750 hbar conduit à une vitesse du son dans le matériau de :

$$
\mathrm{C}=\sqrt{\frac{\mathrm{Eo}}{\rho}}=2000 \mathrm{~m} / \mathrm{s}
$$

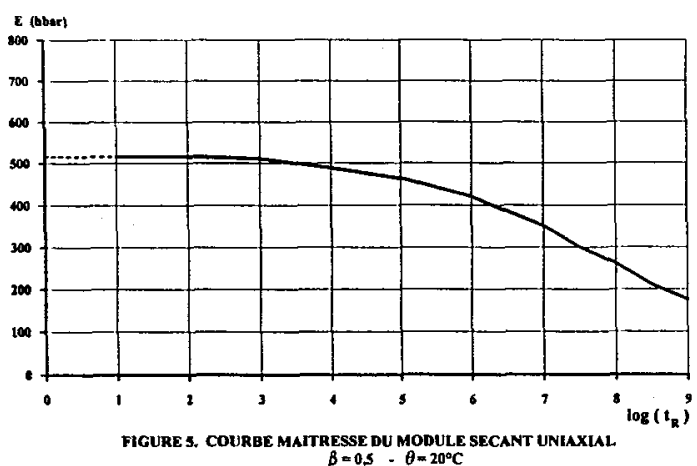
$\beta=0,5-\theta=20^{\circ} \mathrm{C}$

\section{CONTRAinte (tas)}

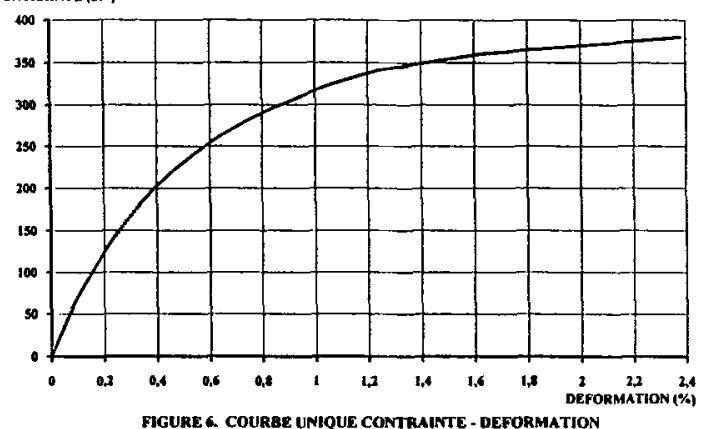
EN COMPRESSION DVNAMHQUE 


\section{DISPOSITIF EXPERIMENTAL (figure $n^{\circ}$ 7)}

Un piston vient impacter un échantillon explosif $\varnothing 10$ - h 20 $\mathrm{mm}$ collé à une enclume en acier. La vitesse du piston est réglable entre 0 et $15 \mathrm{~m} / \mathrm{s}$. L'enclume, d'un diamètre égal à celui de l'échantillon, permet d'obtenir un état de contrainte identique de part et d'autre de l'interface.

La contrainte transmise dans l'enclume en acier est mesurée à l'aide d'une jauge d'extensométrie calibrée et placée à proximité de l'interface échantillon-enclume. La vitesse de l'onde de contrainte, générée par l'impact sur l'échantillon, est relevée par des jauges PVDF.

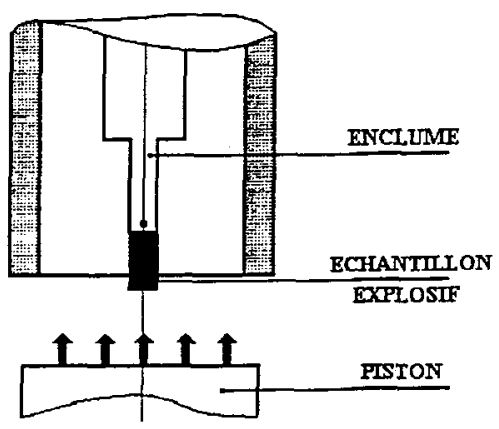

FIGURE 7. DISPOSIIIF EXPERTMENTAL

\section{ANALYSE DE L'ETAT DE CONTRAINTE DANS L'ECHANTILLON EXPLOSIF [2]}

\subsection{Mesures}

Pour une vitesse d'impact de $4,6 \mathrm{~m} / \mathrm{s}$, la figure $n^{\circ} 8$ donne l'évolution de la contrainte transmise dans l'enclume et par conséquent celle dans l'explosif. Cet enregistrement met en évidence l'accroissement progressif, par l'association de rampes et paliers, de la contrainte jusqu'à rupture de 1 'explosif.

Les paliers mettent en évidence des compressions successives (et donc des endommagements) résultant des réflexions de l'onde de contrainte aux différentes interfaces lors de sa propagation dans

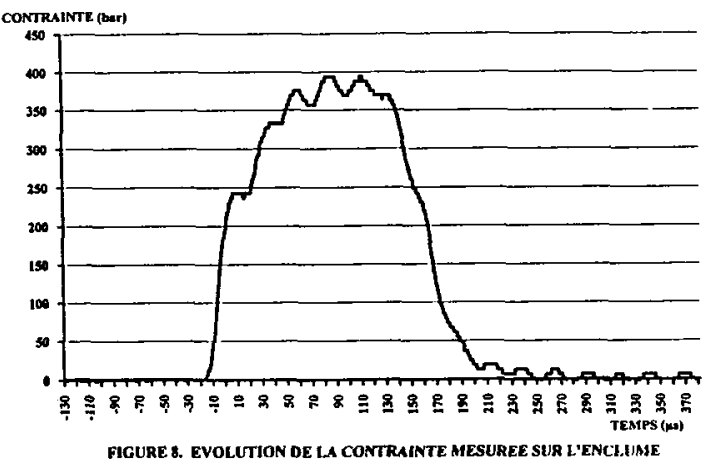
l'échantillon.

Pour la gamme de vitesses considérée, la contrainte de rupture est obtenue, au plus, en $100 \mu \mathrm{S}$ et atteint une valeur comprise entre 350 et 400 bar, en bon accord avec celle prédite par le modele (380 bar).

En outre, la figure 8 montre que, pendant la durée de l'essai, l'état de contrainte n'est pas homogène.

Ainsi la définition de valeurs moyennes de la contrainte et de la déformation n'a aucun sens dans le cas présent. Seules ont une signification les grandeurs locales, notamment à proximité des interfaces.

La vitesse de propagation de l'onde de contrainte, mesurée à $2000 \pm 100 \mathrm{~m} / \mathrm{s}$, est identique à celle prévue par le modèle.

\subsection{Modèle analytique}

Le concept du modèle est fondé sur un parcours de l'onde de contrainte correspondant au nombre d'allers-retours dans l'échantillon nécessaire pour obtenir la rupture (quatre au maximum selon les observations expérimentales). 
La méthode prend en compte :

- les rampes de montée en contrainte,

- la propagation de l'onde de contrainte,

- la non-homogénéité des déformations dans l'échantillon,

- l'endommagement progressif de l'explosif, en particulier au voisinage des interfaces pistonéchantillon et échantillon-enclume,

- l'évolution associée des coefficients de transmission et de réflexion.

L'expression de ces coefficients dépend des sections respectives de l'échantillon et de l'enclume (la même dans notre cas) et des vitesses du son propres à chaque matériau.

La vitesse de propagation des ondes est bien connue dans l'acier. La valeur pour l'explosif endommagé peut être calculée par la relation suivante :

$$
C(\beta)=\sqrt{\frac{E(\beta)}{\rho}}
$$

La densité $\rho$ est supposée constante (des mesures de variations volumiques en quasi-statique ont montré que les variations de $\rho$ étaient de l'ordre de $1 \%$ ).

La méthode de calcul analytique proposée suit les étapes suivantes :

a) L'onde de contrainte incidente, générée par l'impact du piston, crée un premier échelon de déformation dans l'échantillon :

$$
\varepsilon_{1}=\frac{V p}{C o}
$$

Vp est la vitesse du piston et Co est la vitesse du son dans l'explosif sain. On utilise alors la courbe unique contrainte-déformation donnée par le modèle pour déterminer la contrainte $\sigma_{1}$, et déduire la vitesse du son $\mathbf{C}_{1}$ après l'endommagement initial de l'échantillon.

$$
C_{1}=\sqrt{\frac{\sigma_{1}}{\varepsilon_{1} \rho}}
$$

b) A l'autre extrémité de l'échantillon, la réflexion de l'onde génère une variation de la vitesse matérielle $V_{1}$ dans l'explosif. La déformation totale est :

$$
\varepsilon_{2}=\varepsilon_{1}+\frac{V_{1}}{C_{1}}
$$

$\mathrm{V}_{1}$ est déterminée à partir du coefficient de réflexion calculé avec $\mathrm{C}_{1}$ ou déduite de la mesure de la contrainte transmise dans l'enclume.

L'état de contrainte $\sigma_{2}$ et la vitesse du son $C_{2}$, associés à ce nouvel endommagement, sont déduits de la courbe unique contrainte-déformation. $\sigma_{2}$ doit être égale à la contrainte du premier palier mesurée sur l'enclume. La comparaison de ces valeurs est un test de validité du modèle rhéologique utilisé.

c) L'onde réfléchie se déplace jusqu'à l'interface piston-échantillon. Le mouvement du piston impose une vitesse matérielle égale à $\mathrm{V}_{\mathbf{p}}$. La déformation totale est alors :

$$
\varepsilon_{3}=\varepsilon_{2}+\frac{V_{1}}{C_{2}}
$$

Il suffit de reproduire cette procédure pour les trois paliers suivants pour calculer les nouvelles contraintes. 


\section{COMPARAISON RESULTATS EXPERIMENTAUX - MODELE ANALYTIOUE}

Pour chaque palier de contrainte, la figure $n^{\circ} 9$ présente l'évolution des contraintes calculées et expérimentales en fonction de la vitesse du piston.

L'état de contrainte expérimental a été établi pour quatre températures comprises entre 0 et $80^{\circ} \mathrm{C}$. Il a été déterminé en relevant les valeurs correspondant aux paliers enregistrés par la jauge d'extensométrie.

On n'observe aucune évolution significative en fonction de la température, les mesures se situant dans la même dispersion. Celle-ci est de l'ordre de $10 \%$, aux vitesses élevées, pour le premier palier ; elle est beaucoup

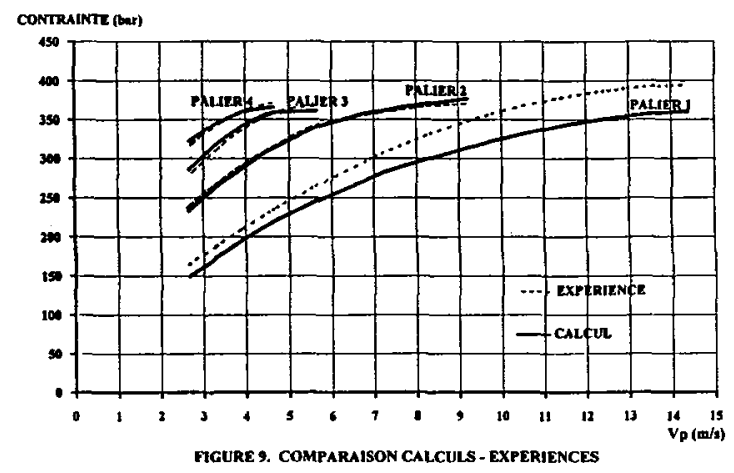
plus faible pour les autres paliers.

Pour tous les paliers et quelle que soit la température, la contrainte maximale mesurée est proche de 380 bar. Remarquons que pour un essai d'une durée de $1 \mathrm{~ms}$ effectué à $80^{\circ} \mathrm{C}$, l'équivalence tempstempérature (figure $n^{\circ} 4$ ) nous conduit à une valeur du temps réduit de $1 \mathrm{~s}\left(\log \mathrm{t} / \mathrm{at} \mathrm{t}_{\theta}\right.$ ) ce qui nous situe dans une région des courbes maîtresses (figures $n^{\circ} 3$ et 4 ) où les propriétés n'évoluent pas en fonction de la vitesse et de la température. Ce résultat est cohérent avec les observations expérimentales précédemment décrites.

La comparaison entre les valeurs expérimentales et les courbes théoriques déterminées par le modele montre que l'approche du problème est satisfaisante. La loi quasi-statique est donc apte à dócrire le comportement de l'explosif dans le domaine du dynamique moyen.

\section{REFERENCES}

[1] BELMAS R., REYNIER P.

Mechanical behavior of pressed explosives.

International Symposium on Energetic Materials Technology - ORLANDO (USA) - 1994.

[2] ROUSSEAU C.

Comportement dynamique en compression uniaxiale d'un explosif agrégataire.

Thèse CNAM - TOURS (France) - 1994. 